\title{
Primordial non-Gaussianity and diffuse Galactic emissions
}

\author{
Alessandro Renzi* \\ SISSA, Trieste \\ University of Padua, Padua E-mail: alexnino@sissa.it
}

\begin{abstract}
We study the bispectrum of astrophysical foregrounds as a potential contaminant of measurements of primordial non-Gaussianity. Namely, we measure the strength of the non-Gaussian signal in terms of a bispectrum amplitude parameter $f_{\mathrm{NL}}$ and evaluate the contribution to $f_{\mathrm{NL}}$ coming from diffuse galactic emission. In our analysis we implement a Skew-Cl estimator, which allows to characterize the contributions to $f_{\mathrm{NL}}$ as a function of scale in harmonic space. We analyse WMAP-like simulations including foreground templates for WMAP's Q, V and W frequency bands. We measure the effect of foregrounds on the estimator variance, as well as the presence of potential bias and the characteristic signatures of residual contamination (after masking the data) in harmonic space.
\end{abstract}

Big Bang, Big Data, Big Computers,

September 19-21, 2012

Laboratoire Astroparticule et Cosmologie, 10 rue A. Domon et L. Duquet, 75205 Paris 13, France

${ }^{*}$ Speaker. 


\section{Introduction}

Emissions from our Galaxy are a limiting factor for most Cosmic Microwave Background (CMB) studies. Given the highly non-Gaussian and anisotropic nature of galactic foreground, this is particularly true for studies related to the search of CMB primordial non-Gaussianity (NG) from inflaton.

In this article we investigate the effect of contamination of Galactic foregrounds on the primordial non-Gaussian parameter $f_{\mathrm{NL}}$. This parameter is a measure of deviation from a Gaussian distribution of the primordial curvature perturbations [1]. It basically measures the amplitude of 3-point function of the CMB temperature fluctuation in harmonic space, called the angular bispectrum.

Due to rotational invariance of the CMB sky, the primordial angular bispectrum is different from zero only in correspondence to triples of wavenumbers that form closed triangles in harmonic space. Different inflationary models make specific predictions about the functional dependence of the bispetrum on different triangles [13]. We can thus construct theoretical bispectrum templates that match a specific primordial theory and fit them to the data in order to obtain a model dependent measurement of the amplitude parameter $f_{\mathrm{NL}}$ (see e.g. [2] and references therein). The most important theoretical bispectrum templates, encompassing a large number of modes, are the so called called local, equilateral and orthogonal templates; by construction they are all factorizable in harmonic space as the product of three separate functions of each wavenumber; this is an important property to ensure feasibility of the numerical analysis. A more detailed description of these issues, as well as the most recent limits on $f_{\mathrm{NL}}$ for these templates can be found in [6].

In this work we will focus on the local primordial template (that is shown by previous studies to be the most contaminated by foreground emission) and we will consider potential contamination from the following Galactic foregrounds [4]: free-free emission, arising from electron-ion scattering; synchrotron emission, arising from the acceleration of cosmic ray electrons in the Galactic magnetic field; and dust emission, arising from thermal emission of large grains of dust in our Galaxy.

Our study will be based public available WMAP satellite data.We will consider in particular the three "cosmological" channels Q, V and W (respectively at 41, 61 and $94 \mathrm{GHz}$ ). From the physics of emissions in Galaxy we know that $\mathrm{W}$ should be mostly contaminated by dust, while synchrotron is expected to dominate in the $\mathrm{Q}$ and $\mathrm{V}$ bands. Our aim is firstly to employ realistic simulations in order to check whether the recovered $f_{N L}$ mean value and error bars are affected by the presence of foregrounds. Moreover we also want to quantify the extent of the contamination as well as characterize possible foreground dependent bispectrum signatures that may allow to separate the spurious signal from the primordial one. After training our estimator on synthetic maps we will then study possible foreground bispectrum contamination in actual WMAP data.

This kind of study is not new in the literature. The WMAP team already provides, for example, an estimate of the effect of Galactic foregrounds in $f_{\mathrm{NL}}$ studies [6]. They compare the $f_{\mathrm{NL}}$ estimated from the optimal combination of $\mathrm{V}$ - and $\mathrm{W}$-band foreground-reduced maps with the $f_{\mathrm{NL}}$ estimated from the same clean map but marginalized over the synchrotron, free-free, and dust foreground templates. Considering the local shape bispectrum they find $f_{\mathrm{NL}}^{\text {local }}=41 \pm 21$ for the clean map and $f_{\mathrm{NL}}^{\text {local }}=31 \pm 21$ for the marginalized map concluding that Galactic foreground emission gives 
rise to a contamination effect of $\Delta f_{\mathrm{NL}}^{\text {local }} \sim 10$. For equilateral and orthogonal templates the effect seems smaller and it is quantified in $\Delta f_{\mathrm{NL}}^{\text {equi }} \sim-3$ for equilateral and $\Delta f_{\mathrm{NL}}^{\text {ortho }} \sim-4$ for orthogonal. In this work we want to expand on previous analyses using a specific bispectrum estimator, the so called "skew-Cl" estimator. The peculiarity of the skew-Cl statistics is that it reduces the level of data compression in harmonic space, thus allowing to extract from the data not just a single $f_{\mathrm{NL}}$ amplitude, but an 1-dependent function. The slope of this function is expected to vary for different theoretical and foreground induced bispectra, thus allowing a better separation of the various contribution as well as the development of useful diagnostic tests. In this preliminary analysis we focus on the primordial local bispectrum template, although we plan on extending the analysis also to equilateral and orthogonal.

The scheme of this manuscript is as follows. In section 2 we summarize our simulation set-up. In section 3 we describe the estimator that will be used for our NG studies. In section 4 provide details on the different sky masks used for this work, and on how they were realised. Finally we will show the $f_{N L}$ and skew-Clsignatures coming from the galactic foreground templates. We then conclude summarising our main results and discussing future developments and plans.

\section{Sky simulations}

The foregrounds templates used in this work are consistent with those used in [9].

We construct all sky CMB maps at different frequencies including the effect of three main diffuse Galactic emission mechanisms: the synchrotron emission, using the data by [14]; we including space varying spectral indices (following [15]) obtained from analyses of the WMAP team [4]; the dust total intensity is based on the analysis of IRAS and DIRBE data by [16], implementing model 8 of frequency scaling and including spatial variations of dust frequency scaling; the free-free emission, traced by $\mathrm{H} \alpha$ emission, has also been included.

We simulate sky maps with foreground emissions and CMB signal at $\mathrm{Q}, \mathrm{V}$ and $\mathrm{W}$ frequencies; we include beam and instrumental noise for each channel using specifications and inputs provided by the WMAP team ${ }^{1}$. To simulate the CMB signal we adopt a $\Lambda$ CDM cosmological model with WMAP 7yr cosmological parameters [18]. We choose HEALPix ${ }^{2}$ parameter Nside $=512$ and $1_{\max }=1000$. We then estimate $f_{\mathrm{NL}}$ using the Skew-Cl algorithm described in the following section.

\section{The skew-Cl $f_{\mathrm{NL}}$ estimator}

The skew-Cl estimator was originally proposed in [3]. It is a bispectrum based statistics [11], inspired by the WMAP team point-like $f_{\mathrm{NL}}$ estimator [10]. One of its advantage with respect to previous approaches is that besides providing an $f_{\mathrm{NL}}$ estimate, it also measures the contribution to $f_{\mathrm{NL}}$ as a function of scale in harmonic space; this lower level of data compression is particularly useful in presence of contribution to the bispectrum coming from different components (roughly speaking, the idea is to measure "as many NG amplitudes as components" and jointly fit for all the bispectra in the data. In order to construct the estimator, the basic idea is to start from a well suited set of filtered maps (with filters depending on the primordial model under study). Cubic

\footnotetext{
${ }^{1}$ http://lambda.gsfc.nasa.gov/

${ }^{2}$ Hierarchical Equal Area isoLatitude Pixelization, http://healpix.jpl.nasa.gov
} 
combinations of this maps are then built (still according to model-dependent prescriptions), and the power spectrum of the cubic combinations ("skew-Cl" or "bispectrum related power spectrum") is finally extracted.

The filtered maps are constructed as

$$
\begin{aligned}
A(\hat{\mathbf{n}}, r) & \equiv \sum_{l m} b_{l} \alpha_{l}(r)\left(C^{-1} a\right)_{l m} Y_{l m}(\hat{\mathbf{n}}), \\
B(\hat{\mathbf{n}}, r) & \equiv \sum_{l m} b_{l} \beta_{l}(r)\left(C^{-1} a\right)_{l m} Y_{l m}(\hat{\mathbf{n}}), \\
C(\hat{\mathbf{n}}, r) & \equiv \sum_{l m} b_{l} \gamma_{l}(r)\left(C^{-1} a\right)_{l m} Y_{l m}(\hat{\mathbf{n}}), \\
D(\hat{\mathbf{n}}, r) & \equiv \sum_{l m} b_{l} \delta_{l}(r)\left(C^{-1} a\right)_{l m} Y_{l m}(\hat{\mathbf{n}}),
\end{aligned}
$$

in which $b_{l}$ is the experimental beam, $\mathrm{C}$ is the total power spectrum including the CMB signal, $C_{l}^{C M B}$, and noise, $N_{l},\left(C_{l} \equiv C_{l}^{C M B} b_{l}^{2}+N_{l}\right)[12] ;$ and

$$
\begin{aligned}
& \alpha_{l}(r)=\frac{2}{\pi} \int k^{2} d k g_{T l}(k) j_{l}(k r), \\
& \beta_{l}(r)=\frac{2}{\pi} \int k^{2} d k P_{\Phi}(k) g_{T l}(k) j_{l}(k r), \\
& \gamma_{l}(r)=\frac{2}{\pi} \int k^{2} d k P_{\Phi}^{1 / 3}(k) g_{T l}(k) j_{l}(k r), \\
& \delta_{l}(r)=\frac{2}{\pi} \int k^{2} d k P_{\Phi}^{2 / 3}(k) g_{T l}(k) j_{l}(k r),
\end{aligned}
$$

where $g_{T l}$ is the temperature radiation transfer function ${ }^{3}$ and $P_{\Phi}$ is the primordial curvature perturbation power spectrum.

The power spectrum of the filtered maps is defined as

$$
\begin{aligned}
C_{l}^{X, Y Z} & =\int d r r^{2} \frac{1}{2 l+1} \sum_{m} \operatorname{Real}\left\{X_{l m}(r)(Y Z)_{l m}(r)\right\}, \\
(Y Z)_{l m}(r) & =\int d \hat{\Omega} Y(r, \hat{\Omega}) Z(r, \hat{\Omega}) Y_{l}^{m}(\hat{\Omega}),
\end{aligned}
$$

where $\mathrm{X}, \mathrm{Y}, \mathrm{Z} \in\{\mathrm{A}, \mathrm{B}, \mathrm{C}, \mathrm{D}\}$ defined above.

Following the arguments in [3] we can now write the Skew-Cl estimator for the three shapes as

$$
\begin{aligned}
C_{l}^{\text {local }}= & \frac{1}{3}\left(C_{l}^{\mathrm{A}, \mathrm{BB}}+2 C_{l}^{\mathrm{B}, \mathrm{AB}}\right)-\left(C_{l}^{\mathrm{A},<\mathrm{BB}>}+2 C_{l}^{\mathrm{B},<\mathrm{AB}>}\right), \\
C_{l}^{\mathrm{equi}}= & -\left(C_{l}^{\mathrm{A}, \mathrm{BB}}+2 C_{l}^{\mathrm{B}, \mathrm{AB}}\right)+2\left(C_{l}^{\mathrm{B}, \mathrm{CD}}+C_{l}^{\mathrm{C}, \mathrm{DB}}+C_{l}^{\mathrm{D}, \mathrm{BC}}\right)-2 C_{l}^{\mathrm{D}, \mathrm{DD}}+ \\
& 3\left(C_{l}^{\mathrm{A},<\mathrm{BB}>}+2 C_{l}^{\mathrm{B},<\mathrm{AB}>}\right)-6\left(C_{l}^{\mathrm{B},<\mathrm{CD}>}+C_{l}^{\mathrm{C},<\mathrm{DB}>}+C_{l}^{\mathrm{D},<\mathrm{BC}>}\right)+ \\
& 6 C_{l}^{\mathrm{D},<\mathrm{DD}>}, \\
C_{l}^{\text {ortho }}= & -3\left(C_{l}^{\mathrm{A}, \mathrm{BB}}+2 C_{l}^{\mathrm{B}, \mathrm{AB}}\right)+6\left(C_{l}^{\mathrm{B}, \mathrm{CD}}+C_{l}^{\mathrm{C}, \mathrm{DB}}+C_{l}^{\mathrm{D}, \mathrm{BC}}\right)-8 C_{l}^{\mathrm{D}, \mathrm{DD}}+ \\
& 9\left(C_{l}^{\mathrm{A},<\mathrm{BB}>}+2 C_{l}^{\mathrm{B},<\mathrm{AB}>}\right)-18\left(C_{l}^{\mathrm{B},<\mathrm{CD}>}+C_{l}^{\mathrm{C},<\mathrm{DB}>}+C_{l}^{\mathrm{D},<\mathrm{BC}>}\right)+ \\
& 24 C_{l}^{\mathrm{D},<\mathrm{DD}>} .
\end{aligned}
$$

\footnotetext{
${ }^{3}$ Obtained from a modified version of CAMB code, http://camb.info
} 
In the formula 3.11, 3.12 and 3.13, MC denotes Monte-Carlo averages over CMB simulations including all experimental features like noise, beam, masking etc. The MC averages appear in terms that are linear in the $a_{l m}$ 's, so that they are zero on average and they do not bias the cubic statistics. The linear term is necessary to make the estimator optimal in presence of statistically anisotropic terms in the data, in particular sky cut and anisotropic noise.

Considering the estimated CMB bispectrum

$$
\hat{B}_{l l_{1} l_{2}}=\sum_{m m_{1} m_{2}}\left(\begin{array}{lll}
l & l_{1} & l_{2} \\
m m_{1} m_{2}
\end{array}\right) a_{l m} a_{l_{1} m_{1}} a_{l_{2} m_{2}}
$$

we can finally construct the Skew-Cl estimator as

$$
C_{l}^{\text {shape }}=\frac{f_{\mathrm{NL}}^{\text {shape }}}{6(2 l+1)} \sum_{l_{1}} \sum_{l_{2}}\left\{\frac{B_{l l_{1} l_{2}}^{\text {shape }} \hat{B}_{l l_{1} l_{2}}}{C_{l} C_{l_{1}} C_{l_{2}}}\right\},
$$

where $B_{l l_{1} l_{2}}^{\text {shape }}$ is the bispectrum for the considered $f_{\mathrm{NL}}$ model (i.e. local in the present study). Note that if we sum over $\sum_{l}(2 l+1)$ we also recover the point-like $f_{\mathrm{NL}}$ estimator of [10], so that the skew-Cl statistics can be seen as an extension of the standard $f_{\mathrm{NL}}$ estimators.

\section{Foreground $f_{\mathrm{NL}}$}

In this section we show the analysis performed by applying the Skew-Cl estimator to WMAP data in the Q, V and W channels. In our analysis we adopt the KQ75 mask described in [7]. That is recommended mask for NG WMAP studies.

As an example, the Galactic emission for the $\mathrm{V}$ channel according to our foreground templates is showed in figure 1 .

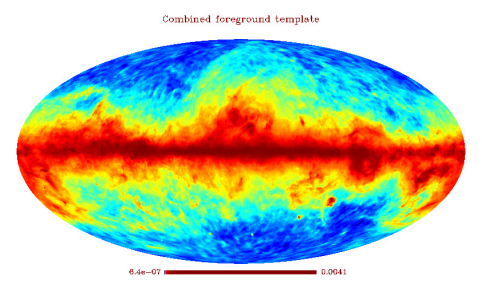

Figure 1: This galactic template map is obtained as the sum of the main Galactic foreground template: free-free, synchrotron and dust, and smoothed at the angular resolution of WMAP V-channel. Units are dimensionless and we adopt a logarithmic scale color in order to make the foreground contribution visible als at high latitudes.

We first validate our pipeline by applying the Skew-Cl estimator to 600 CMB Gaussian realizations with KQ75 mask and for the three channels, including the local shape only and without any foreground. Results are shown in Figure 2. Plots of Skew-Cl show an increase of scatter and error bars at high-ell when the data are noise dominated.

To quantify contamination from foregrounds, we take the same 600 simulations as above, and we add to them the foreground templates previously described. Skew-Cl plots are shown in Figure 3 and the estimated means $f_{N L}^{\text {local }}$ are shown in Table 1.

A by-eye inspection of the plots in Figure 3 already shows that even with the KQ75 mask we still have residual foreground contamination in our results. This effect is very visible on both the low and the high $\ell$ values. Quantitatively this effect is shown in Table 1 . The contamination does not seem to bias the recovered values of $f_{N L}^{\text {local }}$ (except for the most synchrotron contaminated Q 


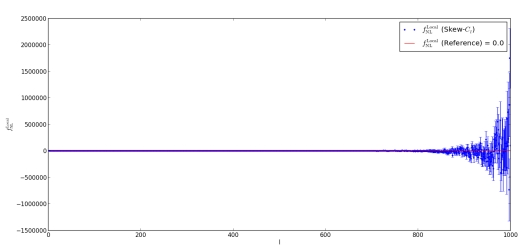

(a) Gaussian reference, channel Q, local shape (b) Gaussian reference, channel Q, local shape, low-ell zoom

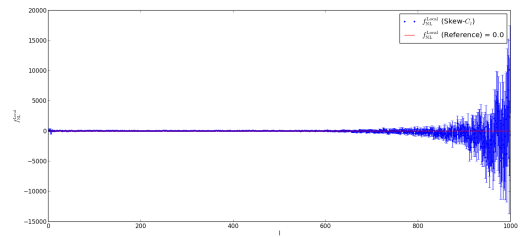

(c) Gaussian reference, channel V, local shape
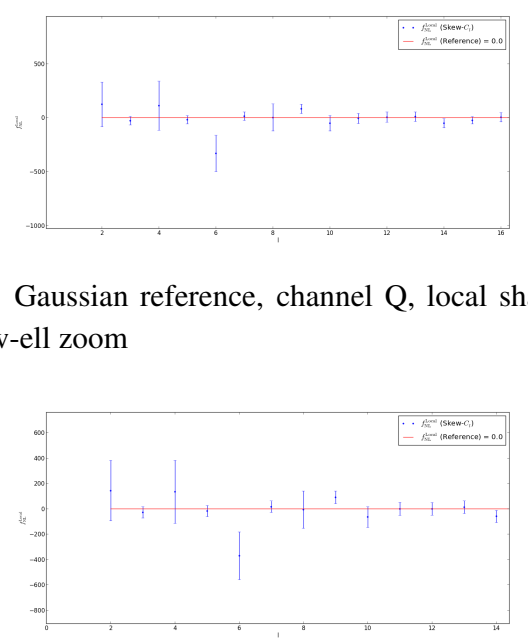

(d) Gaussian reference, channel V, local shape, low-ell zoom
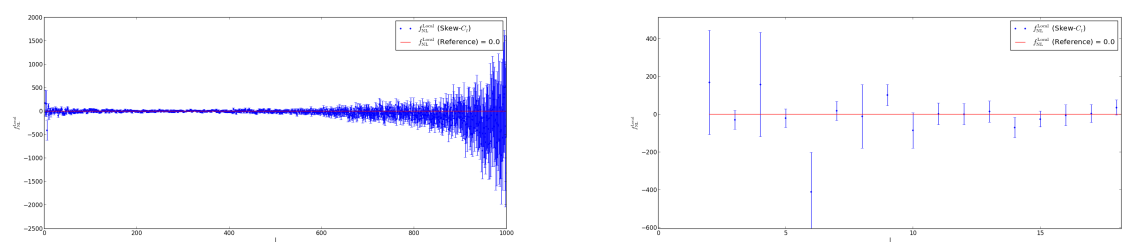

(e) Gaussian reference, channel W, local shape (f) Gaussian reference, channel W, local shape, low-ell zoom

Figure 2: Skew-Cl plots for the channels Q, V and W for local shapes. Error bars (1-sigma) are estimated from $600 \mathrm{CMB}$ Gaussian realizations. Right plots are a zoom of the lowest ells.

Table 1: Estimate of foreground induced $f_{\mathrm{NL}}^{\text {local }}$ vs Gaussian reference for the Q, V and W WMAP7 channels.

$\begin{array}{ccc}\text { Channel } & \text { Reference }\left\langle f_{\mathrm{NL}}^{\text {local }}\right\rangle_{600 \text { maps }} & \text { Template added }\left\langle f_{\mathrm{NL}}^{\text {local }}\right\rangle_{600 \text { maps }} \\ \mathrm{Q} & -1.2 \pm 29.5 & -13.3 \pm 33.1 \\ \mathrm{~V} & -0.8 \pm 25.5 & -1.7 \pm 26.2 \\ \mathrm{~W} & -0.7 \pm 25.7 & -2.1 \pm 26.3\end{array}$

channel). However the table shows an effect on the variance that is increased with respect to the Gaussian reference case. These results are consistent with previous findings from the WMAP team and other groups [8].

\section{Conclusion}

In this preliminary analysis we show how the galactic foregrounds emission can potentially contaminate measurement of $f_{\mathrm{NL}}^{\text {local }}$. The effect of residual foregrounds contamination should be 


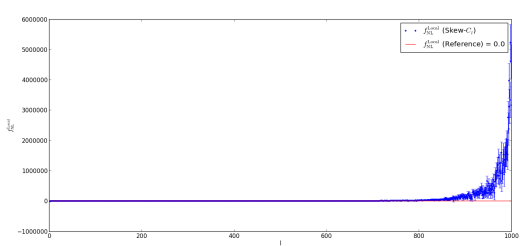

(a) Gaussian reference + template, channel Q, local shape

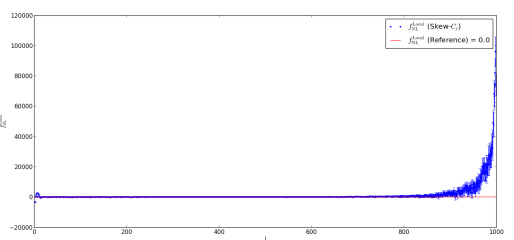

(c) Gaussian reference + template, channel V, local shape

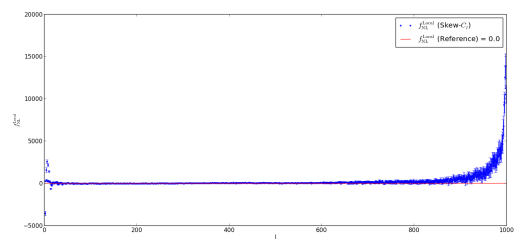

(e) Gaussian reference + template, channel W, local shape

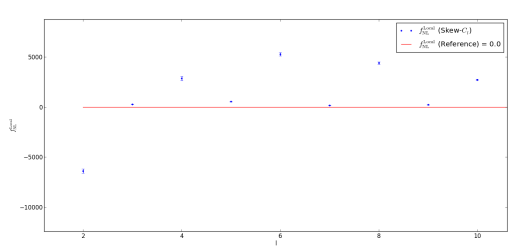

(b) Gaussian reference + template, channel Q, local shape, low-ell zoom

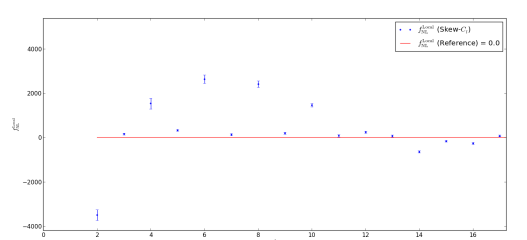

(d) Gaussian reference + template, channel V, local shape, low-ell zoom

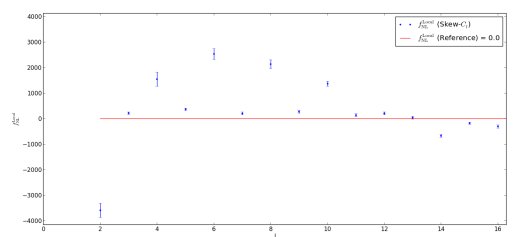

(f) Gaussian reference + template, channel W, local shape, low-ell zoom

Figure 3: Skew-Cl plots for the channels Q, V and W for local shapes. Error bars (1-sigma) are estimated from $600 \mathrm{CMB}$ Gaussian realizations to which we sum the foreground template. Right panels are a zoom of the lowest ells.

seriously taken into account in view of more sensitive forthcoming CMB dataset, such as the one that will be provided by the ESA Planck satellite. For the future we plan to extend this analysis to all the bispectrum shapes an all the WMAP channels and look for the effect on separated foregrounds templates (free-free, synchrotron and dust). We also plan on using our foreground skew-Cl slopes extracted from simulations to perform a full joint analysis of primordial shapes and spurious bispectra.

Some of the results in this paper have been derived using the HEALPix [17] and CAMB packages [19].

\section{References}

[1] E. Komatsu Class. Quant. Grav. 27 (2010) 124010 [arXiv: 1003.6097 ]

[2] M. Liguori, E. Sefusatti, J. R. Fergusson, E. P. S. Shellard Advances in Astronomy 2010 (2010) 980523 [arXiv:1001.4707]

[3] D. Munshi and A. Heavens MNRAS 401 (2010) 2406 [arXiv: 0904 . 4478] 
[4] C. L. Bennett et al. ApJS 148 (2003) 97B [astro-ph/0302208]

[5] E. Komatsu and D. N. Spergel Phys.Rev. D63 (2001) 063002 [arXiv:astro-ph/ 0005036 ]

[6] E. Komatsu et al. ApJS 192 (2011) 18 [arXiv: 0803 . 0547]

[7] B. Gold et al. ApJS 192 (2011) 15 [arXiv: 1001 . 4555]

[8] K. M. Smith, L. Senatore and M. Zaldarriaga JCAP 0909 (2009) 006 [arXiv: 0901.2572 ]

[9] E. Jeong, C. Baccigalupi and G. F. Smoot JCAP. 09 (2010) 018 [arXiv: 1004.1046 ]

[10] E. Komatsu, D. N. Spergel and B. D. Wandelt ApJ 634 (2005) 14 [astro-ph/ 0305189 ]

[11] D. Babich Phys.Rev. D 72 (2005) 043003 [astro-ph/ 0503375 ]

[12] A. P. S.Yadav, E. Komatsu, B. D. Wandelt, M. Liguori, F. K. Hansen and S. Matarrese ApJ 678 (2008) $578[\operatorname{arXiv}: 0711.4933]$

[13] N. Bartolo, E. Komatsu, S. Matarrese and A. Riotto Phys. Rept. 402 (2004) 103 [astro-ph/0406398]

[14] C. G. T. Haslam, C. J. Salter, H. Stoffel and W. E. Wilson Astron. Astrophys. Suppl. Ser. 47 (1982) 1

[15] G. Giardino, A. J. Banday, K. M. Górski, K. Bennett, J. L. Jonas and J. Tauber A\&A 387 (2002) 82

[16] D. P. Finkbeiner, M. Davis and D. J. Schlegel ApJ 524 (1999) 867

[17] K. M. Górski et al. ApJ 622 (2005) 759 [astro-ph/ 0409513 ]

[18] D. Larson et al. Astron. Astrophys. Suppl. Ser. 192 (2011) 16 [arXiv: 1001 . 4635]

[19] A. Lewis, A. Challinor and A. Lasenby ApJ 538 (2000) 473 [astro-ph/9911177] 